Farum

Sociológico

\section{Forum Sociológico}

Série II

$27 \mid 2015$

Mobilidade científica \& imigração qualificada

\title{
La movilidad social y geográfica de las mujeres de ciencia
}

\author{
Gabriela de la Peña Astorga
}

\section{Q OpenEdition}

1 Journals

Edição electrónica

URL: https://journals.openedition.org/sociologico/1313

DOI: 10.4000/sociologico.1313

ISSN: 2182-7427

Editora

CICS.NOVA - Centro Interdisciplinar de Ciências Sociais da Universidade Nova de Lisboa

\section{Edição impressa}

Data de publição: 21 dezembro 2015

Paginação: 79-81

ISSN: 0872-8380

\section{Refêrencia eletrónica}

Gabriela de la Peña Astorga, «La movilidad social y geográfica de las mujeres de ciencia», Forum Sociológico [Online], 27 | 2015, posto online no dia 27 maio 2016, consultado o 31 março 2022. URL: http://journals.openedition.org/sociologico/1313 ; DOI: https://doi.org/10.4000/sociologico.1313 


\title{
LA MOVILIDAD SOCIAL Y GEOGRÁFICA DE LAS MUJERES DE CIENCIA
}

\author{
Gabriela de la Peña Astorga \\ - Universidad Autónoma de Coahuila, Facultad de Ciencias de la Comunicación
}

Me llamo Gabriela de la Peña Astorga y tengo 43 años. Soy profesora investigadora de tiempo completo en la Facultad de Ciencias de la Comunicación de la Universidad Autónoma de Coahuila; resido en la ciudad de Saltillo, Coahuila, México. Me dedico a la investigación, a la docencia y a la acción social orientadas al desarrollo participativo y equitativo de mi entorno, que es complejo y plural como no puede dejar de serlo cualquier sociedad contemporánea. Me considero parte de una generación de científicas sociales mexicanas caracterizadas por hacer camino en la ciencia bajo las condiciones que más adelante explicaré.

Me han invitado a preparar para este número de Forum Sociológico, un testimonio de mi experiencia de movilidad como mujer de ciencia. Lo hago a continuación rescatando algunas reflexiones que he realizado a lo largo de estos veinte años de construcción de identidad profesional, que, como estoy segura de que le ha sucedido a las mujeres que han abrazado a la ciencia como un llamado ineludible a lo largo de todos los tiempos, es para mí una actividad permanente y constituye la "doble reflexividad" (de investigadora y de mujer) de la que hablan tan amplia y profundamente mis colegas y amigas en Portugal, Beatriz Padilla y Thais França.

Van entonces estas líneas a continuación, en un formato de escritura que agradezco se me haya permitido utilizar de forma menos rígida que las normas a las que tenemos que ceñirnos los investigadores -hombres y mujeres- cada vez que entregamos públicamente el resultado de nuestro trabajo. Sin afán de protagonismo, intentaré deconstruir las condiciones bajo las que hoy puedo dedicarme de "tiempo completo" (el título de mi contrato laboral) a la formación de otros y otras, y a la curiosidad sistematizada con "la ley a mi favor" (Parcialmente. Me refiero a la conquista de las mujeres por tener acceso a la educación universitaria y a la contratación como profesoras investigadoras; aunque aún queda un largo camino por recorrer en este campo para las actuales y futuras generaciones). Pido de antemano una disculpa por la omisión que sin duda haré de la situación de otras mujeres de ciencia en el mundo, pero también sé que los lectores de este testimonio podrán identificarse con algunas de las reflexiones que aquí verteré: ninguna persona es una isla, y las condiciones estructurales de desequilibrio, desafortunadamente, son casi siempre las mismas para casi todos en una época determinada de nuestra historia.

\section{Llegar a la universidad, primera movilidad geográfica y social}

En México, según datos de la OCDE (2014) el año 2014, sólo un 30\% de los jóvenes de 20 años se encuentran matriculados como estudiantes en algún plantel del sistema universitario en México. De ellos, el $49.35 \%$ son mujeres (INEGI, 2014). En el año 1989, cuando inicié mis estudios de licenciatura, a nivel nacional la razón entre mujeres y hombres que ingresaban a la universidad era de .749 mujeres. Para el estado de Coahuila, era de .755 (INEGI, 2015). Casi el $50 \%$ de dichos jóvenes, éramos y son mujeres, lo que constituye un primer dato esperanzador para la equidad de género en materia de acceso a la educación superior; razón por la cual apoyo las políticas de cuota en ésta y otras áreas de desarrollo y representatividad para cualquier sociedad. No obstante estos porcentajes, entre hombres y mujeres la elección de carrera se ve permeada por otras condiciones, no siempre visibles y no siempre conscientes para unos y otros. "Estudia comunicación, es una carrera muy bonita para ti como mujer", me dijo mi padre con toda la honestidad y el amor de su corazón. Afortunadamente, dicha sugerencia estuvo en consonancia con lo que yo deseaba para mi futuro profesional. Afortunadamente, yo provenía de una familia que consideró importante mi formación escolar desde la infancia y que se había hecho cargo del gasto económico y de los recursos necesarios para que yo pudiera culminar mi educación básica y media superior. Afortunadamente, pude elegir matricularme en una institución de educación superior en una ciudad cercana a la mía, para cumplir el anhelo que muchas niñas tienen desde pequeñas: mudarme de ciudad, dejar el nido familiar, construir una biografía llena de experiencias de lo más diversas. Afortunadamente. No ha sido así ni sigue siendo de esta forma para las 3, 860000 jóvenes mexicanas de 15 a 19 años de edad (el 70\% de ellas) que para el año 2013 no ingresaron al sistema universitario (INMUJERES, 2014). 
Elegir a la(s) ciencia(s) social(es) como camino profesional, segunda movilidad geográfica y social

Era mi penúltimo semestre en la licenciatura. Había ya explorado varios campos profesionales de la carrera de comunicación. El primer doctor en comunicación de masas que arribaba a mi facultad, recién graduado de una universidad extrajera ${ }^{1}$, me mostraba algo que yo vislumbré como "lo mío", una aproximación que integraba los saberes y las prácticas a las que, sin saber que podía existir un camino profesional para ello, siempre había querido dedicarme. Movilidad para mí, era entonces, llevar y traer saberes y metodologías a otras, muchas, partes del mundo y de mi entorno; una vida dedicada exclusivamente, con permiso y con facilidades de crecimiento laboral, a la INVESTIGACIÓN (trato de reproducir la fascinación que esta palabra despertó en mi persona a los 21 años de edad). Por ello, y como resultado de los trabajos que después he realizado en el campo de la educomunicación, propongo de manera tan insistente una investigación-acción participativa que incluya como herramienta de acción y como aproximación teórica al Aprendizaje por Modelamiento, de Albert Bandura (1982). ¿Había camino para las mujeres en esta actividad? El acercamiento a las asociaciones profesionales de investigadores en México, así como la revisión de literatura científica hecha por mujeres, me indicaba que sí. ¿Era posible para mí llegar a ser una investigadora social? Posible, sí. Fácil, no. Para el año 1990 , sólo el $21 \%$ de los investigadores registrados en el $\mathrm{SNI}^{2}$, eran mujeres. Para 1998, esta cifra aumentó al $28 \%$, aunque para el año 2000 se redujo este porcentaje y aumentó el de la participación de los hombres en comparación con la de las mujeres (Pérez Sedeño y otros, 2006). También para 1994, las mujeres inscritas en algún programa de posgrado representaban el $37.4 \%$ de los estudiantes de este nivel educativo en el país (INEGI, 2015). Estudié la Maestría en Ciencias con especialidad en Comunicación Internacional (19941997) con la esperanza de cumplir con los requisitos laborales que se me solicitaban para entrar a un salón de clase como maestra, para poder recibir un sueldo como investigadora e iniciar la larga y tortuosa carrera (según escuchaba decir a mis profesores -en su mayoría hombres- y profesoras) de convertirme en una persona de ciencia (nunca a nadie le escuché decir "mujer de ciencia", ni entonces ni después). Mi beca consistía en laborar parcialmente en el área de relaciones públicas del Tecnológico de Monterrey y cursar la maestría al mismo tiempo ( $y$ atender casa y matrimonio). Terminado el posgrado, pude ingresar a un aula como profesora. Renuncié a mi trabajo como coordinadora de comunicación escrita en la Universidad Virtual de la misma institución (después de dar todas las explicaciones correspon- dientes por estar rechazando un trabajo "estable" para aventurarme desde cero en otro) y descubrí que para poder tener un puesto de trabajo en ello, de tiempo completo, era necesario realizar un doctorado. Siguiente parada: Universidad de Barcelona, Doctorado en Antropología Social y Cultural. ¿Beca de doctorado? No había elegido un programa que fuera parte de las prioridades de desarrollo del país; tampoco era profesora de planta (contrato fijo), que era un requisito para recibir apoyo de la universidad en la cual laboraba. Ya en España en el año 2000, que "seguía empeñada en estudiar el programa que ya me habían dicho que no era prioritario para México y para las agencias de cooperación internacional en ese momento" (a pesar de mis notas sobresalientes como estudiante en la UB y de las sentidas cartas de recomendación elaboradas por mis profesores de éste y del anterior programa de posgrado), fue la razón que me dieron para negarme nuevamente una beca como estudiante mexicana en el extranjero; con otras palabras, ésta fue la misma respuesta que recibí de otras instituciones de apoyo a estudiantes de doctorado (una beca para mujeres, entonces, ini soñar! Simplemente, no existían). Trabajar y estudiar, y recibir el apoyo económico que mi familia podía ofrecerme a la distancia, fue para mí, como para muchos estudiantes de doctorado nacionales y extranjeros, una realidad que por compromiso moral, considero profundamente en el abordaje de los problemas de investigación-acción y en las gestiones académicas que ahora realizo; y que, sin duda, me llevaron a elegir las líneas de investigación en las que hoy desarrollo mi trabajo.

\section{Participar en redes científicas internacionales, tercera movilidad geográfica y social}

¿Qué hace posible la movilidad internacional de las mujeres de ciencia? La respuesta podría componerse de múltiples factores. Desde mi punto de vista, el primero es haber superado las dos movilidades a las que hago referencia más arriba; que se dicen rápido, pero cuya meta conlleva para toda mujer establecer estrategias en el terreno para crecer en medio de grandes y cotidianas condiciones adversas, y que por falta de espacio, resulta imposible mencionar aquí. El segundo, tiene que ver con la visibilidad de su trabajo, exigencia establecida como indicador en la evaluación de su producción científica y, como podemos observar a través de los datos proporcionados sobre el ejercicio de la ciencia como profesión en México, tampoco constituye un campo favorecedor o equitativo para ellas (nosotras). Considero que esta tercera movilidad (conquista de, en realidad) consiste en, finalmente, poder ser puentes entre y constructoras con otros (nunca solas, eso lo hemos aprendido bien: nada se logra negando la solidaridad 
con otras vulnerabilidades; a nivel local, nacional o internacional) de conocimientos y experiencias que han nacida de una niña que imaginaba un mundo diferente en el cual ella pudiera desarrollarse (no me refiero - sólo - a mí misma, sino a los millones de niñas en el mundo que se supieron y se saben desposeídas de unos derechos cuyo ejercicio debería ser equitativo y que les es negado por una irracional lógica patriarcal - una locura institucionalizada - que les resulta incomprensible desde su pura capacidad intelectual y desde el enorme potencial que en ellas mismas reconocen).

\section{Los estudios y las acciones de género, cuarta movilidad social y geográfica}

Tampoco se crea que elegir como línea de investigación y de acción a los estudios de género, es tarea fácil o consecuencia natural para todas las mujeres de ciencia. En el camino de construir su propia propuesta para abordar los problemas científicos, sean sobre mujeres o no, las académicas han tenido que madurar cuestiones relacionadas con su propia identidad como miembros de una sociedad determinada, revisar su propia biografía, cuestionarse la condición de género bajo la que han construido su trayectoria científica, construir un papel particular en la identidad de la profesión que eligieron, sin perder de vista que han sido parte de pequeño porcentaje de sus congéneres que pudo tener acceso a una educación de posgrado y a un reconocimiento por su trabajo académico; y decidir cómo y sobre qué investigar considerando todo lo anterior. No obstante dicha revisión, de la que se podría pensar que la consecuencia automática es que las mujeres de ciencia tengan como prioridad dedicarse al estudio de la falta de equidad de género y abocarse a encontrar sus soluciones, las actuales condiciones de producción científica a nivel mundial, imposibilitan en muchos casos que esto sea así; no es así ni por la violación a la libertad que significaría imponer una línea de investigación a cualquier científica desde ninguna perspectiva hegemónica -así fuera la constituida por una ideología femenina- ni porque se encuentren en ambientes libres de prejuicios contra el trabajo científico de, con y para las mujeres de su entorno y de otros contextos. Por ello, considero que una cuarta movilidad social y geográfica, es aquella que se deriva de la decisión y de las estrategias establecidas por las mujeres de ciencia, para incluir en su trabajo de investigación y de acción social a una perspectiva de género aplicada a los temas de su interés.
Cuatro movilidades sociales y geográficas que pueden considerarse, hoy por hoy, cuatro conquistas de las mujeres de ciencia.

Por cierto, tengo como hábito no colgar etiquetas a ningún grupo social o persona, pero me parece que esta de "mujer de ciencia" para mis colegas científicas, representa por mi parte un reconocimiento a su trayectoria, una contestación al tradicional modelo patriarcal de la actividad científica que sigue operando mayormente en todos los países, y un abrazo solidario a las mujeres que hoy, a pesar de todo, siguen eligiendo a la ciencia como camino profesional.

\section{Notas}

1 Me refiero al Dr. José Carlos Lozano, que había egresado del Doctorado en Periodismo y Comunicación de Masas de la Universidad de Texas en Austin. Amigo, mentor y mi director de tesis de la Maestría en Comunicación del Tecnológico de Monterrey, Campus Monterrey. Quisiera dar cuenta en este momento de cuan inspirador fue su ejemplo, durante su trayectoria como profesor investigador en dicha institución, para muchos estudiantes de licenciatura y posgrado.

2 Sistema Nacional de Investigadores, padrón que integra a los investigadores mexicanos que han cumplido con los requisitos de producción científica en alguno de sus 5 niveles (candidato, I, II, III y hemérito) establecidos por el Consejo Nacional de Ciencia y Tecnología (CONACYT).

\section{Referencias bibliográficas}

BANDURA, A. (1982), Teoría del aprendizaje social, Madrid, Espasa-Calpe.

INEGI (2014), Matrícula y procesos escolares en México. (19 de septiembre de 2014) Obtenido de http:// www3.inegi.org. $\mathrm{mx} /$ sistemas/sisept/default. aspx? $\mathrm{t}=$ medu $17 \& \mathrm{~s}=\mathrm{est} \& \mathrm{c}=21788$

INEGI (2015), Indicadores de desarrollo de los objetivos del milenio. (10 de octubre de 2015)

Obtenido de: http://www.inegi.org.mx/prod_serv/ contenidos/espanol/bvinegi/productos/nueva_ estruc/HyM2014/23.\%20IndicadoresODM.pdf

INMUJERES (2014), Mujeres y hombres en México 2014. (24 de enero de 2014) Obtenido de http://www. inmujeres.gob.mx/inmujeres/images/frontpage/ redes_sociales/myh_2014.pdf

OCDE (2014), Panorama de la educación 2014. (10 de octubre de 2015). Obtenido de http://www. oecd.org/edu/Mexico-EAG2014-Country-Note-spanish.pdf

PÉREZ SEDEÑO, E. et al. (2006), Ciencia, tecnología y género en Iberoamérica, Madrid, Consejo Superior de Investigaciones Científicas.

Recebido a 11/10/2015. Aceite para publicação a 06/12/2015.

Gabriela de la Peña Astorga (gabriela.pena@uadec.edu.mx). Universidad Autónoma de Coahuila, Facultad de Ciencias de la Comunicación. Carretera a Zacatecas Km. 3 s/n, Centro, 25000 Saltillo, Coahuila, México. 